Dra. Blanca I. Vega Castro, CPA*

\title{
Las normas internacionales de contabilidad y su efecto en las fusiones y adquisiciones
}

\author{
*Profesora de Contabilidad \\ Universidad de Puerto Rico en Ponce \\ Correo electrónico: blancav@ coqui.net \\ Recibido: 22 de agosto de 2006 \\ Aceptado: 14 de noviembre de 2006
}

\section{Summary}

The main objective of this paper is to investigate whether the international accounting harmonization has favored the globalization process. This investigation examined the relationship between international accounting standards and the international merger and acquisition activities in Argentina, Brazil, Chile, Mexico, Peru and Venezuela from 1995 to 2004. This research tested four hypotheses and used analytics such as panel data analysis, correlation, nonparametric tests and logistic regression.

Key word: accounting; harmonization; merger and acquisition; globalization.

\section{Resumen}

Con el fin de conocer si la armonización contable internacional es un mecanismo que favorece el proceso de globalización, se examinó la relación entre la adopción de normas internacionales de contabilidad y la incidencia de actividades de fusiones y adquisiciones internacionales que se llevaron a cabo desde 1995 a 2004 en Argentina, Brasil, Chile, México, Perú y Venezuela. Se establecieron cuatro hipótesis y se utilizaron diversos instrumentos analíticos como datos de panel, correlación, pruebas no paramétricas y regresión logística.

Palabras clave: contabilidad; armonización; fusiones y adquisiciones; globalización.

Introducción

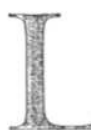

a inversión extranjera directa (IED) se considera una manifestación evidente del proceso de globalización de la economía.

Según un informe del Banco Mundial (2002), la más reciente etapa de la globalización, que comenzó a principios de los años ochenta, se caracteriza por la determinación de algunos países - principalmente los menos desarrollados - en mejorar sus climas de inversión y abrirse al comercio exterior. De acuerdo con la Conferencia de las Naciones Unidas sobre Comercio y Desarrollo (UNCTAD, 2003), en el ámbito mundial durante el período 1991-2002, de los 1,641 cambios en las leyes que rigen las IED, el 95\% instituyó un marco más favorable a las inversiones foráneas. De los hallazgos de la UNCTAD surge que continuarán los esfuerzos por atraer IED como parte de una competencia mundial cada vez mayor.

FORUM EMPRESARIAL 12, 1 (mayo, 2007): 3-21

ISSN 1541-8561 
Los beneficios de la IED en el crecimiento económico del país receptor son mencionados por autores como Aitken, Harrison y Lipsey, 1996; Blomstrom y Kokko, 1997; Borensztein, De Gregorio y Lee, 1998; Feldstein, 2000; Findlay, 1978; Hymer, 1960; Kolstad y Villanger, 2004 entre otros. Las formas más comunes de IED son las fusiones y adquisiciones (FAS) internacionales. Éstas representaron el $83 \%$ del total mundial de las IED para el 1999, (UNCTAD, 2000).

Moon, Kim y Lee (2002) consideran que las FAS internacionales han contribuido a la competitividad de la economía receptora. Esta situación es particularmente importante para los países en desarrollo, sobre los que Balasubramanyan, Salisu y Sapsford, 1996; Bengoa y Sánchez, 2000; Blomstrom y Kokko, 1996; De Gregorio, 1992; y Hansen y Rand, 2004 demuestran la influencia positiva y significativa en el crecimiento económico. Muchas entidades empresariales, tanto en el ámbito nacional como internacional, han escogido a las FAS como estrategias de crecimiento dado que representan el mecanismo más rápido para conquistar una posición sólida en un mercado nuevo, ganar poder en el mismo, incrementar el tamaño de la empresa y diluir los riesgos (UNCTAD, 2000).

A finales del siglo pasado y durante el actual, se han llevado a cabo numerosos estudios y se han desarrollado diferentes teorías y modelos que tratan de dar una explicación a las motivaciones de los inversionistas para invertir en el extranjero (Dunning, 1998; Hymer, 1960; Seyf, 2001; Vernon, 1974). Éstos parecen coincidir en que tanto las características internas de las empresas transnacionales como las del país receptor son elementos determinantes en la elección del modo de inversión en el extranjero. A pesar de los numerosos estudios que contrastan las condiciones y características de los países receptores de caudales extranjeros examinados en la revisión de literatura, ninguno contempla las prácticas de contabilidad como criterio al seleccionar el lugar para la inversión en el extranjero.

Las economías nacionales están hoy más dispuestas a aceptar la inversión extranjera a la par que los inversionistas y sus compañías buscan oportunidades más allá de sus fronteras. Sin embargo, la inversión en un mercado global representa un gran reto para las empresas debido a que se enfrentan a circunstancias completamente nuevas. A lo largo y ancho del mundo existen diferencias culturales $4 \quad$ ISSN 1541-856

FORUM EMPRESARIAL 12, 1 (Mayo, 2007) profundas y complejas, también las hay en la forma de determinar y presentar la información financiera de las empresas.

Existen divergencias entre los países en cuanto al formato y la terminología de los estados financieros, pero las diferencias más significativas son las discrepancias en las normas de valoración de los activos y pasivos y la determinación del ingreso. Por ejemplo, en los Estados Unidos la plusvalía se trata como un activo, mientras que en el Reino Unido constituye una reducción del capital (Wolk, Dodd y Tearney, 2004). Así mismo, a las compañías estadounidenses no se les permite presentar los activos en el estado de situación por una cuantía mayor que su costo histórico, mientras que las compañías en México y Chile valoran sus activos por un precio ajustado por la inflación y ciertas compañías holandesas lo divulgan por el costo de reemplazo. Finalmente, cabe señalar como diferencias que en los Estados Unidos, los costos de investigación y desarrollo deben registrarse como gastos según se incurran, mientras en Canadá y Francia pueden ser capitalizados como activos. Diferencias como las mencionadas y muchas otras, generan resultados significativamente desiguales en los estados financieros.

El caso de la compañía Daimler-Benz, actualmente DaimlerChrysler, es un ejemplo clásico para ilustrar cuán significativas pueden ser estas diferencias. En 1993, cuando ésta decidió convertirse en la primera empresa alemana en cotizar en el New York Stock Exchange tuvo que reconciliar sus estados financieros con las normas de contabilidad en cumplimiento con el Formulario 20-F. Bajo los principios de contabilidad de Alemania, Daimler había obtenido ganancias millonarias (DM615 millones), pero de acuerdo con los principios de contabilidad generalmente aceptados de Estados Unidos (US GAAP, por sus siglas en inglés), la compañía incurrió en una pérdida tres veces mayor (DM1,800 millones) (Flower, 2002). Ante esta disparidad, sólo es posible imaginar la confusión y preocupación de los inversionistas que toman decisiones financieras confiando en la información de la contabilidad.

Para ese mismo año, la Comisión de Valores e Intercambio de Estados Unidos (SEC, por sus siglas en inglés) publicó un estudio que examina las reconciliaciones con los principios de contabilidad estadounidenses generalmente aceptados hechas por 444 empresas extranjeras de 36 países (SEC, 1993). Los resultados del estudio indi- 
caron que aproximadamente dos terceras partes de las compañías extranjeras devengaron un ingreso neto significativamente menor según las normas estadounidenses. Es decir, los resultados financieros de las empresas van a depender de las normas de contabilidad que se utilicen para valorar los activos y pasivos, y reconocer los ingresos y gastos. Como puede observarse, las diferencias entre las normas de contabilidad -sin emitir juicio sobre cuáles son las mejoresafectan la comparación o uniformidad contable de los informes financieros.

La Junta de Normas Internacionales de Contabilidad (IASB, por sus siglas en inglés) es una de las organizaciones internacionales que está trabajando para lograr la armonización internacional de las normas de contabilidad (Nobes y Parker, 2002). Durante más de treinta años, la IASB y su antecesor, el Comité de Normas Internacionales de Contabilidad (IASC, por sus siglas en inglés), han trabajado con el propósito de formular y promulgar las normas de contabilidad para la presentación de los estados financieros y promover su aplicación y aceptación mundial. Las normas internacionales de contabilidad existentes (NICs) y las nuevas normas internacionales de información financiera (NIIFs) se encuentran en constante evolución. Para el 2004, se habían emitido cinco NIIFs y cuarenta y una NICs, algunas de las cuales se han revisado.

A pesar de que el IASB no puede obligar a los países a adoptar sus normas, el grado de aceptación internacional de sus pronunciamientos ha ido en aumento. Esta aceptación se manifiesta en los acuerdos con la Organización Internacional de Comisiones de Valores (IOSCO, por sus siglas en inglés) -organismo que dirige las bolsas de valores en el ámbito mundial-y en disposiciones del Fondo Monetario Internacional, del Banco Mundial y de las Naciones Unidas, entre otras entidades. En el 2000, la IOSCO recomendó que los organismos reguladores del mundo permitan que los emisores multinacionales utilicen las normas internacionales al elaborar sus estados financieros para emisiones y colocaciones en la bolsa de valores. Hay otros adelantos en el proceso de uniformidad, como la recomendación de la Comisión Europea de que a partir de 2005 se le exija a las empresas que cotizan en la bolsa de valores aplicar las normas internacionales en la preparación de sus estados financieros consolidados (Flower,

$6 \quad$ ISSN 1541-8561

FORUM EMPRESARIAL 12, 1 (Mayo, 2007)
2002). Quizás el evento más relevante a favor de la armonización de normas contables es la vinculación formal entre los dos organismos contables líderes, la Junta de Normas de Contabilidad Financiera (FASB) y la Junta de Normas de Contabilidad Internacional (IASB). En el año 2002, estos organismos formalizaron el compromiso de lograr la convergencia entre sus normas.

En esta investigación nos proponemos analizar el papel que desempeña la información de contabilidad en la asignación de los recursos, de manera que sean asignados más eficientemente. La misma se propone responder a la siguiente interrogante: ¿La armonización de las normas de contabilidad entre los países, propicia el proceso de globalización?

\section{Metodología}

Se seleccionó una muestra de seis países latinoamericanos: Argentina, Brasil, Chile, México, Perú y Venezuela. Se analizaron las transacciones de fusiones y adquisiciones llevadas a cabo para el periodo de 1995 a 2004 por empresas de estos países latinoamericanos. En la selección de estos países se tomó en cuenta su desarrollo comercial, el cual se determinó utilizando la lista de las primeras cincuenta empresas transnacionales más grandes de las economías en desarrollo ubicadas en Latinoamérica, según el World Investment Report. Se utilizó este indicador porque se considera una forma de medir el desarrollo económico en estos países y su grado de exposición a la economía global.

Se seleccionaron dos variables independientes; el grado de adopción de las Normas Internacionales de Contabilidad y el efecto del riesgo-país. Éste es el riesgo que encaran los inversionistas extranjeros cuando invierten en un país específico, comparado con la alternativa de invertir en otro. Estas variables independientes se relacionaron con las actividades de fusiones y adquisiciones que fue la variable dependiente.

La información necesaria para esta investigación se obtuvo de diferentes fuentes. Los datos sobre las actividades de fusiones y adquisiciones se obtuvieron de un banco de datos recopilado especialmente para esta investigación por la compañía Thompson. Dicha fuente compila las transacciones de fusiones y adquisiciones llevadas a cabo en Ar- 
gentina, Brasil, Chile, México, Perú y Venezuela durante el periodo 1995-2004. Estos datos incluyen el origen de las empresas involucradas en estas transacciones, distinguiendo entre adquiriente-quienes provienen de todas partes del mundo-y adquirido. También incluyen el volumen y la frecuencia de estas transacciones en los países seleccionados durante los años bajo estudio. El número total de fusiones y adquisiciones realizadas, de acuerdo con esta base de datos, asciende a 1,996 .

Los datos sobre el riesgo de dichos países para el periodo 1995-2004 provienen de la publicación mensual International Country Risk Guide (ICRG, Guía internacional sobre el riesgo de países), publicada por Political Risk Services (PRS), una empresa de servicios privada e internacional. Esta empresa emplea analistas que proporcionan clasificaciones del riesgo mediante un sistema basado en un conjunto de 22 componentes agrupados en tres categorías de riesgo: político, financiero y económico. A cada componente se le asigna un valor numérico máximo, de cero a cien, en el cual un mayor número de puntos indica el riesgo más bajo y el menor número el riesgo más alto (el riesgo más alto $=0$ y el más bajo $=100$ ).

Los datos sobre la emisión de las normas internacionales anuales se obtuvieron de la Junta de Normas Internacionales de Contabilidad (IASB). En cuanto a las normas de los países se obtuvieron a través de los organismos profesionales y gubernamentales de cada uno de los países bajo estudio.

El grado de adopción de las normas internacionales de contabilidad para cada país por año se obtuvo examinando y comparando las normas internacionales con las normas nacionales. Esencialmente, el análisis se limitó a la comparación de las normas publicadas por los organismos que establecen las normas en cada uno de los países en la muestra. No se consideraron los pronunciamientos de contabilidad que en algunas ocasiones son incluidos en otros medios normativos profesionales, tales como los referentes a auditoria, código de ética profesional o algún otro. Todo el procedimiento se estableció con el fin de ubicarse en la esencia de las normas y lograr compararlas de la mejor manera posible.

\section{Hallazgos}

Antes de examinar los resultados del análisis de las hipótesis propuestas, es conveniente resumir los valores de cada una de las variables estudiadas en el periodo analizado.

\section{Promedios variables estudiados}

A continuación, la Tabla 1 presenta los promedios de las variables analizadas:

Tabla 1

Promedios de variables estudiadas en el periodo 1995-2004

\begin{tabular}{|lcccc|}
\hline \multicolumn{1}{|c}{ País } & $\begin{array}{c}\text { Por ciento } \\
\text { adopción }\end{array}$ & Riesgo-País & $\begin{array}{c}\text { Por ciento } \\
\text { Frecuencia }\end{array}$ & $\begin{array}{c}\text { Por ciento } \\
\text { Volumen }\end{array}$ \\
\hline Argentina & 25.8 & 68.3 & 47.6 & 61.5 \\
\hline Brasil & 39.3 & 64.9 & 42.8 & 47.9 \\
\hline Chile & 55.4 & 77.4 & 44.8 & 47.5 \\
\hline México & 70.8 & 70.3 & 41.9 & 54.3 \\
\hline Perú & 81.6 & 66.8 & 52.4 & 67.4 \\
\hline Venezuela & 30.9 & 64.2 & 50.7 & 69.4 \\
\hline
\end{tabular}

Según se observa en la Tabla 1, el por ciento promedio de adopción por país fluctuó de $26 \%$ a $82 \%$. El mayor grado de adopción para el periodo se refleja en las normas de Perú con $81.6 \%$, mientras que el menor grado de adopción lo tiene Argentina con 25.8\%. El por ciento promedio de fusiones y adquisiciones internacionales con respecto al total de fusiones y adquisiciones en cuanto a frecuencia fue $47 \%$; es decir, casi la mitad de las transacciones de fusiones y adquisiciones involucró empresas internacionales que buscaron expandir sus operaciones en Latinoamérica durante el periodo estudiado. Se destacan Perú y Venezuela con más de $50 \%$ de fusiones y adquisiciones internacionales. La proporción menor de fusiones y adquisiciones internacionales del total de fusiones y adquisiciones para el periodo analizado la tiene México con $41 \%$. El volumen de transacciones de fusiones y adquisiciones internacionales alcanzó una 
proporción significativa ( $58 \%$ ) del volumen o monto en dólares del total de transacciones de fusiones y adquisiciones en los países observados. Venezuela, Perú y Argentina reflejan que más del $60 \%$ del volumen de las transacciones de fusiones y adquisiciones provino de fuentes extranjeras.

\section{Grados de adopción de Normas Internacionales de Contabilidad}

La Gráfica 1, a continuación, ilustra los grados de adopción de las normas internacionales en los países en la muestra durante los diez años.

\section{Gráfica 1}

Grados de adopción de las Normas Internacionales de Contabilidad

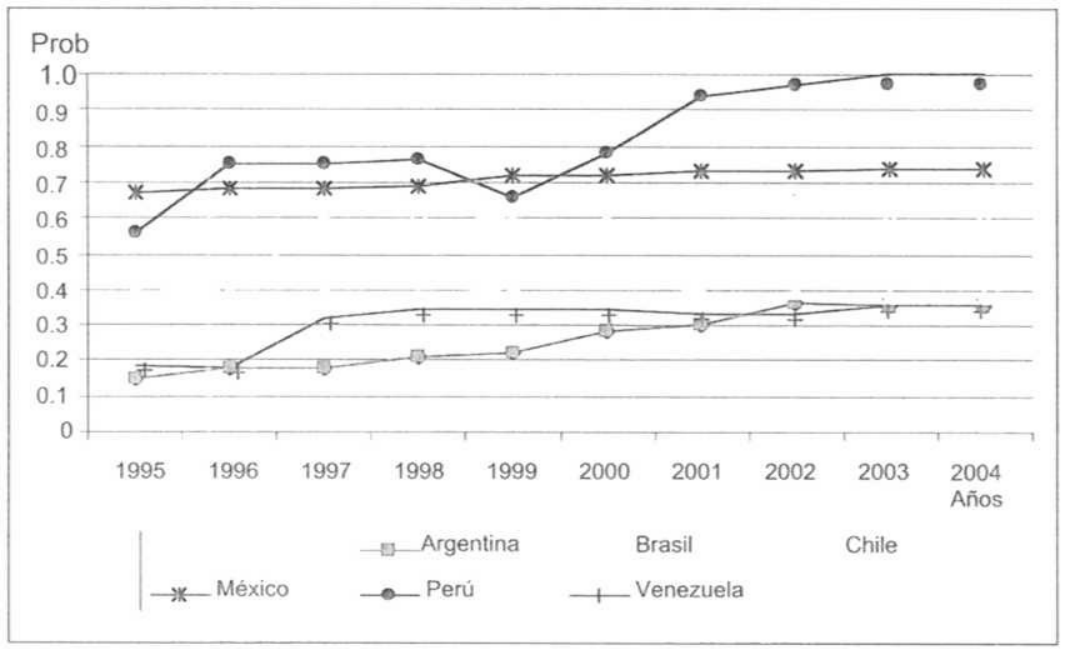

En la Gráfica 1 se puede apreciar que todos los países aumentaron su grado de adopción de 1995 a 2004. Perú y México han intercambiado liderazgo en cuanto a la adopción de Normas Internacionales de Contabilidad. Sin embargo, el primero se ha mantenido a la vanguardia desde el año 2000. Otro país que a partir de 1996 ha mantenido un nivel sostenido por encima del $50 \%$ de adopción ha sido Chile. Los otros tres países, Brasil, Venezuela y Argentina han mantenido un grado de adopción de menos del $50 \%$ con una ten-

10 ISSN 1541-8561 FORUM EMPRFSARIAL 12, I (Mayo, 2007) dencia por parte de Argentina de aumento en el grado de adopción desde niveles por debajo del $20 \%$ en 1997.

Relación entre el grado de adopción de las normas internacionales de contabilidad y la frecuencia en las transacciones de fusiones y adquisiciones internacionales

La primera hipótesis plantea que: Existe relación directa entre el grado de adopción de las normas internacionales de contabilidad y la frecuencia en las transacciones de fusiones y adquisiciones internacionales. Los resultados después de aplicar la metodología de datos de panel, confirman este planteamiento.

Tabla 2

Resultados de la regresión con datos de panel entre la variable dependiente frecuencia de fusiones y adquisiciones internacionales y las variables independientes

\begin{tabular}{|l|c|c|c|}
\hline \multicolumn{1}{|c|}{ Variable } & Coeficiente & Estadística t & Valor $\mathbf{p}$ \\
\hline$\%$ Adop & 0.652 & $(2.46)$ & 0.017 \\
\hline Riesgo & 0.868 & $(2.06)$ & 0.044 \\
\hline $\mathrm{R}=0.575$ & & & \\
\hline
\end{tabular}

De acuerdo con los resultados, el grado de adopción de las normas internacionales de contabilidad contribuye positiva (coeficiente igual a 0.652 ) y significativamente (valor $\mathrm{p}=0.017$ ) a explicar la frecuencia de las transacciones de fusiones y adquisiciones internacionales, tal y como se propone en la primera hipótesis. Este modelo explica el $57.5 \%\left(\mathrm{R}^{2}=0.575\right)$ de la variabilidad en la frecuencia en las transacciones de fusiones y adquisiciones internacionales.

Además, de acuerdo con los resultados, el riesgo del país resulta significativo (valor $\mathrm{p}=0.044$ ) y, por lo tanto, contribuye a explicar la frecuencia de las transacciones de fusiones y adquisiciones internacionales. Existe una relación inversa significativa entre el riesgo-país y la frecuencia de fusiones y adquisiciones internacionales (un valor más alto de este índice representa un riesgo país menor). El modelo 
que contiene tanto las variables grado de adopción, como la de riesgopaís tiene un alto poder explicativo de la conducta de las actividades de fusiones y adquisiciones. A continuación se presentan los resultados de la correlación por país:

Tabla 3

Coeficiente de correlación Pearson para cada país estudiado entre el por ciento de fusiones y adquisiciones internacionales y el grado de adopción de las normas internacionales

\begin{tabular}{|l|c|}
\hline \multicolumn{1}{|c|}{ País } & Correlación \\
\hline Argentina & -0.338 \\
\hline Brasil & 0.528 \\
\hline Chile & 0.509 \\
\hline México & 0.566 \\
\hline Perú & 0.610 \\
\hline Venezuela & -0.381 \\
\hline
\end{tabular}

Como se puede observar en la tabla anterior, con excepción de Argentina y Venezuela, existe una correlación positiva mayor de $50 \%$ entre el grado de adopción de las normas internacionales de contabilidad y la cantidad de fusiones y adquisiciones internacionales. Perú experimentó la correlación más alta $(61 \%)$ entre el grado de adopción y frecuencia de fusiones y adquisiciones internacionales.

Relación entre el grado de adopción de las normas internacionales de contabilidad y el volumen (monto en dólares) de las transacciones de fusiones y adquisiciones internacionales

La segunda hipótesis plantea que: Existe relación directa entre el grado de adopción de las normas internacionales de contabilidad y el volumen (monto en dólares) de las transacciones de fusiones y adquisiciones internacionales. Los resultados a continuación, luego de aplicar la Metodología de Datos de Panel, confirman este planteamiento.
Tabla 4

Resultados de la regresión con datos de panel entre la variable dependiente volumen y las variables independientes grado de adopción (\%Adop) y riesgo del país

\begin{tabular}{|l|c|c|c|}
\hline Variable & Coeficiente & Estadística t & Valor $\mathbf{p}$ \\
\hline$\%$ Adop & 1.492 & $(4.60)$ & 0.000 \\
\hline Riesgo & 0.331 & $(0.459)$ & 0.648 \\
\hline $\mathrm{R}^{2}=0.461$ & & & \\
\hline
\end{tabular}

De acuerdo con los resultados obtenidos, el grado de adopción de las normas internacionales de contabilidad resulta estadísticamente significativo (valor $\mathrm{p}=0.00$ ) y contribuye positivamente a explicar el volumen de las transacciones en fusiones y adquisiciones internacionales, tal y como se propone en la segunda hipótesis. Este modelo explica el $46 \%\left(\mathrm{R}^{2}=.461\right)$ de la variabilidad en el volumen en las transacciones de fusiones y adquisiciones internacionales. La relación entre grado de adopción y volumen de las transacciones de fusiones y adquisiciones es mayor que en el caso de grado de adopción y frecuencia. El coeficiente de la variable riesgo país tiene un valor positivo; sin embargo, no es estadísticamente significativo (valor $\mathrm{p}=0.648$ ). A continuación se presentan los resultados de la correlación por país:

\section{Tabla 5}

Coeficiente de correlación Pearson para cada país estudiado entre el volumen y el grado de adopción de las normas internacionales de contabilidad

\begin{tabular}{|l|c|}
\hline \multicolumn{1}{|c|}{ País } & Correlación \\
\hline Argentina & 0.300 \\
\hline Brasil & 0.713 \\
\hline Chile & 0.909 \\
\hline México & 0.724 \\
\hline Perú & 0.676 \\
\hline Venezuela & -0.335 \\
\hline
\end{tabular}


Como se puede observar en la Tabla 5, en Argentina, Brasil, Chile, México y Perú existe una correlación positiva entre el grado de adopción de las normas internacionales de contabilidad y en las transacciones de fusiones y adquisiciones internacionales con respecto al volumen. En esta ocasión, sólo Venezuela vuelve a tener una correlación negativa, esta vez en su volumen de transacciones de fusiones y adquisiciones.

Las actividades de fusiones y adquisiciones internacionales entre los países que tienen mayor grado de adopción de las normas internacionales de contabilidad y los que tienen menor grado de adopción de estas normas

La tercera hipótesis plantea que: La internacionalización de los países con niveles bajos de adopción de normas internacionales de contabilidad es menor que la internacionalización de los países con niveles altos de adopción. Los resultados a continuación, después de aplicar la prueba de comparación de Mann-Whitney, confirman esta hipótesis.

Tabla 6

Prueba de Mann-Whitney de diferencias de grupos de adopción

CI: X_2, $\mathrm{Y}_{-} 1$
$\mathrm{X} \_2 \quad \mathrm{~N}=29 \quad$ Mediana $=\quad 39.73$
$\mathrm{Y} \_1 \quad \mathrm{~N}=31 \quad$ Mediana $=\quad 52.17$
Punto estimado para ETA1-ETA2 $=\quad-10.42$
95.1 Por ciento CI para ETA1-ETA2 $=(-17.51,-2.10)$
$\mathrm{W}=722.5$
Prueba de ETAl = ETA2 vs ETA1 < ETA2 es significativa al 0.0084

En este caso el valor es $\mathrm{p}=0.0084$, por lo que se rechaza la hipótesis nula $(\mathrm{p}<0.05)$. La prueba de Mann Whitney sugiere que la mediana de frecuencia de transacciones internacionales del grupo de países con menor grado de adopción es menor que la mediana de la frecuencia de transacciones internacionales de los países con mayor grado de adopción.

La probabilidad de fusiones y adquisiciones internacionales y el grado de adopción de las normas internacionales

La cuarta hipótesis plantea que: A mayor grado de adopción de las normas internacionales, las fusiones y adquisiciones que involucran empresas internacionales son más probables que las que involucran empresas nacionales. Los resultados a continuación, después de aplicar el modelo de regresión logística, confirman este planteamiento.

Tabla 7

Resultados de la regresión logística para $\mathrm{Y}$

\begin{tabular}{|l|c|c|c|}
\hline \multicolumn{1}{|c|}{ Variable } & Coeficiente & Wald & Valor $\mathrm{p}$ \\
\hline Constante & -1.5897 & -2.2331 & 0.0255 \\
\hline \%Adop & 0.0286 & 2.2097 & 0.0271 \\
\hline
\end{tabular}

A la variable fusiones y adquisiciones (variable dependiente), se le asignaron valores binarios: 1 si hubo fusión o adquisición que involucró una empresa extranjera con una local y 0 si hubo fusión o adquisición entre dos empresas locales. La prueba estadística indica que el coeficiente de \% Adop es positivo y significativamente diferente de cero (valor $\mathrm{p}=0.0271$ ) a un nivel de 0.05 . El $62.5 \%$ de las veces el modelo predice correctamente las fusiones y adquisiciones nacionales y el modelo predice correctamente las fusiones y adquisiciones internacionales el $57.1 \%$ de las oportunidades. Los resultados muestran que la variable por ciento de adopción ejerce una influencia positiva y significativa en la probabilidad de transacciones de fusiones y adquisiciones, tal y como lo proponía la hipótesis.

Todos estos hallazgos, estadísticamente fundamentados, conducen a una serie de conclusiones en cuanto al impacto de las normas de contabilidad sobre la globalización. 


\section{Conclusiones}

De los resultados de la investigación se desprenden cinco conclusiones principales:

1. La armonización de las normas de contabilidad contribuye a que aumente la cantidad de fusiones y adquisiciones internacionales.

En primer lugar, se deriva que existe una relación directa entre el grado de adopción de las normas internacionales de contabilidad y la frecuencia en las transacciones de fusiones y adquisiciones internacionales. Es decir, la armonización de las normas de contabilidad contribuye al aumento en la cantidad de fusiones y adquisiciones internacionales. Con excepción de dos países, Argentina y Venezuela, existe una correlación positiva mayor de $50 \%$ entre el grado de adopción de las normas internacionales de contabilidad y la cantidad de fusiones y adquisiciones internacionales. Venezuela se caracterizó por una baja actividad de fusiones y adquisiciones (104 de un total de 1,996), la mayor parte de ellas internacionales, lo que pudo hacer fracasar la correlación evidente en los demás países. En Argentina, la crisis económica pudo ser la causa de la contradicción. Es decir, aunque las autoridades contables argentinas siguieron aumentando la armonización con las normas internacionales, la inestabilidad económica pudo haber disuadido a las empresas multinacionales de invertir allí. Mientras que Perú, que es el país con el mayor grado de adopción de normas internacionales, experimentó la correlación más alta entre el grado de adopción y frecuencia de fusiones y adquisiciones internacionales.

2. La armonización de las normas de contabilidad contribuye a que la cuantía monetaria en las transacciones de fusiones y adquisiciones sea mayor.

La segunda prueba indica que existe una relación directa entre el grado de adopción de las normas internacionales de contabilidad y el volumen (monto en dólares) en las transacciones de fusiones y adquisiciones internacionales, tal y como se propuso en la segunda 16 ISSN 1541-8561 FORUM EMPRESARIAL 12, 1 (Mayo, 2007) hipótesis. La armonización de las normas de contabilidad contribuye a que la cuantía monetaria en las transacciones de fusiones y adquisiciones sea mayor. La vinculación entre el grado de adopción y volumen de las transacciones de fusiones y adquisiciones es mayor que en el caso de grado de adopción y frecuencia, lo que podría implicar que las empresas multinacionales tienen más recursos financieros que exceden las posibilidades de inversiones internas, por lo que tienden a hacer inversiones mucho más cuantiosas. En esta ocasión, sólo Venezuela vuelve a tener una correlación negativa mientras que Argentina presenta una correlación positiva. El hecho de que ahora se refiera al monto en dólares, mientras que anteriormente se refería a frecuencia, significa que las transacciones que involucran fusiones y adquisiciones en Argentina fueron menores en frecuencia, pero de alto monto en dólares.

3. Conclusiones mixtas en relación al riesgo del país.

Otro factor que podría repercutir en la decisión de este tipo de inversión, y que se analizó en esta investigación, es el riesgo del país. El riesgo aludido se refiere a la inestabilidad política, económica y social. Se concluyó que existe una relación inversa entre el riesgo del país y la frecuencia de fusiones y adquisiciones internacionales. Es decir, cuanto mayor fue el riesgo-país, menor fue la proporción de fusiones y adquisiciones. Sin embargo, no se pudo concluir que existiera relación inversa entre el riesgo país y el volumen de las transacciones de fusiones y adquisiciones internacionales. Esto concurre con los resultados de ciertos estudios como el de Grosse y Trevino, 1996, en los que la variable no ha resultado significativa o su significatividad ha sido muy débil.

4. Los países que tienen mayor grado de adopción de las normas internacionales tienen más actividades de fusiones y adquisiciones internacionales.

Se observó también que la frecuencia de transacciones internacionales del grupo de países con menor grado de adopción es menor que la frecuencia de transacciones internacionales de los países con FORUM EMPRESARIAL 12, 1 (mayo, 2007): 3-2 \begin{tabular}{l|l} 
ISSN $1541-8561 \mid$ & 17
\end{tabular} 
mayor grado de adopción. Es decir, los países con mayor grado de adopción de normas internacionales de contabilidad presentan mayores niveles de inversión extranjera.

5. El grado de adopción ejerce influencia positiva en la probabilidad de transacciones de fusiones y adquisiciones.

Por último, los resultados del modelo logit confirman que la variable grado de adopción ejerce una influencia positiva en la probabilidad de transacciones de fusiones y adquisiciones. Es decir, existe una relación directa entre el grado de adopción de normas internacionales y la probabilidad de que las fusiones y adquisiciones involucren empresas internacionales.

Los resultados permiten afirmar que la adopción de las normas internacionales de contabilidad contribuye a una mayor incidencia de actividades de carácter global como las fusiones y adquisiciones internacionales, puesto que las cuatro hipótesis planteadas alcanzaron significación estadística.

\section{Recomendaciones}

Los resultados de esta investigación muestran la necesidad de disenar e implantar planes de armonización para las normas internacionales. Aun cuando internacionalmente se han iniciado esfuerzos encaminados hacia la armonización, las acciones más significativas deberán tomarse de acuerdo con el país, donde los planes de armonización con las normas internacionales necesitan desarrollarse e implantarse. Todos los países bajo estudio demostraron, a través del aumento en la armonización con las normas internacionales, sus esfuerzos en tal sentido aunque algunos muy lentamente. La armonización debe efectuarse sin demora porque de lo contrario la brecha entre los requerimientos nacionales e internacionales aumentará dramáticamente, conforme se emitan normas internacionales.

Las asociaciones profesionales, las que desempeñan un papel importante en la promulgación de las normas contables nacionales, son las llamadas a promover la armonización y a emitir normas que sean compatibles con las de los demás países. También las universidades, a

\footnotetext{
18 ISSN 1541-8561 FORUM EMPRESARIAL 12, I (Mayo, 2007)
}

través de la investigación y la enseñanza, tienen mucho que aportar en el proceso de la armonización contable. La contabilidad cumplirá con el objetivo fundamental de que los estados financieros produzcan información financiera útil para la toma de decisiones económicas, en la medida en que se provea información que pueda entenderse y compartirse internacionalmente. La información financiera, producto de la compilación de las transacciones mediante la contabilidad, cumple un papel significativo por cuanto es y será una de las piezas claves para la toma de decisiones económicas.

$\mathrm{Al}$ adaptar las políticas económicas en el ámbito nacional y regional a las expectativas de los mercados internacionales, Latinoamérica podría atraer flujos mayores de capital. A pesar de la incertidumbre, Latinoamérica sigue siendo muy atractiva para la inversión y las empresas extranjeras no pasarán por alto las oportunidades que brinda este mercado. La opción de las normas internacionales de contabilidad podría contribuir a mejorar la percepción internacional de los países como receptores de inversiones. En un mundo cada vez más integrado e interdependiente, la prosperidad de algunos países depende más que nunca de los resultados económicos que obtengan otros países. 
Bibliografía

Aitken, B.; Harrison, A. \& Lipsey. R. (1996). Wages and Foreign Ownership: A Comparative Study of Mexico, Venezuela, and the United States. Journal of International Economics, 40 (3), 345-71.

Balasubramanyan, V., Salisu, M. \& Sapsford, D. (1996). Foreign Direct Investment and Growth in EP and IS countries. Economic Journal, 106, 92-105.

Banco Mundial (2002). Globalization, Growth and Poverty: Building an Inclusive World Economy. World Bank-Oxford University Press.

Bengoa, M. \& Sánchez, B. (2000). Inversión Extranjera Directa en Latinoamérica:Impacto sobre crecimiento económico. Tercer Encuentro de Economía Aplicada. Universidad de Valencia.

Blomstrom, M., \& Kokko, A. (1996). The Impact of Foreign Investment on Host Countries: A Review of the Evidence. Working Paper. The World Bank.

Blomstrom, M. \& Kokko, A. (1997). Regional Integration and Foreign Direct Investment. National Bureau of Economic Research Working Paper 6019.

Borensztein, E., De Gregorio, J. \& Lee, J. (1998). How does Foreign Direct Investment Affect Economic Growth? Journal of International Economics, 45 (1), 115-135.

De Gregorio, J. (1992). Economic Growth in Latin America. Journal of Development Economics, 39, 58-84.

Dunning, J. (1998). Location and the Multinational Enterprise: A neglected Factor? Journal of International Business Studies, 29(1), 45.

Feldstein, M. (2000). Aspects of Global Economic Integration: Outlook for the Future. National Bureau of Economic Research. Working Paper 7899.

Findlay, R. (1978). Relative Backwardness, Direct Foreign Investment, and the Transfer of Technology: a Simple DynamicModel. Quarterly Journal of Economics, 92, 1-16.

Flower, J. (2002). Global Financial Reporting. Palgrave. New York, NY.
Grosse, R. \& Trevino, L.J. (1996). Foreign Direct Investment in the US: An Analys by Country of Origin. Journal of International Business Studies, 27, 139-155.

Hansen, H. \& Rand, J. (2004). On the Causal Links between FDI and Growth in Developing Countries. Institute of Economics, University of Copenhagen Development Economics Research Group (DERG).

Hymer, S. (1960). The International Operations of National Firms: A Study of Direct Foreign Investment. Tesis doctoral. Cambridge MA: MIT Press.

Kolstad, I. \& Villanger, E. (2004). How does Social Development Affect FDI and Domestic Investment? Michelsen Institute Development Studies and Human Rights CMI Reports. Recuperado en diciembre 2, 2004 de www.cmi.no/public/public.htm

Moon, H.; Kim, H. \& Lee, D. (2003). Cross-Border Mergers E Acquisitions: Case Studies of Korea, China and Hong Kong, China. Asia Pacific Economic Cooperation (APEC) \#203-CT-01.6.

Nobes, C. \& Parker, R. (2002). Comparative International Accounting. (7ma.Ed.) England: Pearson Education Limited.

SEC (1993). United States Securities and Exchange Commission. Survey of Financial Statement Reconciliations by Foreign Registrants, Washington, D.C.

Seyf, A. (2001). Can Globalization and Global Localization Explain Foreign Direct Investment? Japanese Firms in Europe. International Journal of the Economics of Business, 8 (1), 137-153.

UNCTAD (2000). World Investment Report 2000: Cross-border Mergers and Acquisitions and Development. United Nations, Washington.

UNCTAD (2003). A Record year for Liberalizing FDI Laws and Regulations. UNCTAD/PRESS/PR/2003/82,

Vernon, R. (1974). Competition Policy toward Multinational Companies. American Economic Review, 64, 276-282.

Wolk, H., Dodd, J. \& Tearney, M. (2004). Accounting Theory: Conceptual Issues in a Political and Economic Environment. Mason, OH: South-Western. 